\title{
Typologie des systèmes de culture de coton biologique au Bénin
}

\author{
Saturnin AZONKPIN ${ }^{1,2 *}$, Daniel Chèpo CHOUGOUROU ${ }^{2}$, Emile Codjo AGBANGBA ${ }^{2}$, \\ Carline Christelle Joanne SANTOS ${ }^{2}$, Mohamed Mansourou SOUMANOU $^{3}$ et \\ Simplice Davo VODOUHE ${ }^{4}$
}

\begin{abstract}
${ }^{1}$ Centre de Recherches Agricoles - Coton et Fibres (CRA-CF), Institut National des Recherches Agricoles du Bénin (INRAB), 01 PB 175 Cotonou, République du Bénin.

${ }^{2}$ Département de Génie de l'environnement, Laboratoire d'Etude et de Recherche en Biologie Appliquée (LARBA), Ecole Polytechnique d'Abomey-Calavi (EPAC), Université d'Abomey-Calavi (UAC), 01 BP 2009, Cotonou, République du Bénin.

${ }^{3}$ Unité de Recherche en Génie Enzymatique et alimentaire (URGEA), Laboratoire d'Etude et de Recherche en Chimie Appliquée (LERCA), Département de Génie de Technologie Alimentaire (DGTA), Ecole Polytechnique d'Abomey-Calavi (EPAC), Université d'Abomey-Calavi (UAC), 01 BP 2009, Cotonou, République du Bénin.

${ }^{4}$ Faculté des Sciences Agronomiques (FSA), Université d'Abomey-Calavi (UAC), 01 BP 2009, Cotonou, République du Bénin.

*Auteur correspondant ; E-mail: azonsat@yahoo.fr ; Tel: +22995564936
\end{abstract}

\section{RESUME}

La connaissance des caractéristiques des systèmes de culture est un atout pour le développement du secteur agricole. C'est dans ce cadre que cette étude vise à identifier et à caractériser les systèmes de culture pratiqués par les producteurs de coton biologique au Bénin. Pour y parvenir, une enquête a été conduite sur un échantillon de 176 producteurs. L'analyse des données par la Classification Ascendante Hiérarchique a permis d'identifier les systèmes de culture. Leur caractérisation a été faite selon les pratiques culturales, le rendement, leur fréquence dans les Communes et les ethnies. Les résultats obtenus ont permis d'identifier quatre systèmes de culture du coton biologique : le système de culture mécanisée avec traction animale qui n'utilise pas de nouveaux paquets technologiques (S1); le système de culture manuelle qui utilise les légumineuses comme précédent cultural (S2); le système de culture mécanisée avec traction animale qui utilise de nouveaux paquets technologiques (S3) et le système de culture mécanisée avec traction animale qui utilise de nouveaux paquets technologiques mais qui continue avec la pratique de défrichement avec brûlis (S4). Les éléments permettant de distinguer les systèmes sont : préparation du terrain, rotation, précédents culturaux, engrais organiques, plantes insecticides, plantes pièges et cultures associées. Tous les producteurs enquêtés à Glazoué, Kandi et Sinendé ont pratiqué respectivement les systèmes de culture S2, S1 et S4. A Banikoara, 96,67\% des producteurs ont pratiqué le système $\mathrm{S} 3$ contre $3,33 \%$ pour le système $\mathrm{S} 1$. Les producteurs de Ouassa-Péhunco pratiquaient à 77,78\% le système $\mathrm{S} 3$ contre 22,22\% pour le système S1. Les enquêtés des ethnies Adja, Fon et Mahi ont pratiqué à 100\% le système S2. Par contre, tous les Haoussa et les Monkolé ont pratiqué le système S1. Les Gando, Peulh et Bariba ne pratiquaient pas spécifiquement un système. Le système de culture S2 a obtenu le rendement moyen le plus élevé $(885,80 \pm 36,3 \mathrm{~kg} / \mathrm{ha})$. En somme, les systèmes de culture varient selon les Communes et les ethnies. Le système $\mathrm{S} 1$ a été le plus pratiqué par les producteurs.

(C) 2018 International Formulae Group. All rights reserved.

Mots clés: Systèmes de culture, coton biologique, pratiques culturales, Bénin, labour mécanisé. 


\title{
Typology of organic cotton growing systems in Benin
}

\begin{abstract}
Understanding growing systems characteristics is relevant for agricultural development. In this context, the present study seeks to identify and characterize the growing systems practised by the producers of Organic Cotton in Benin. To reach this goal, a survey was conducted on a sample of 176 producers. The analysis of the data by Hierarchical classification allowed to identify the growing systems. Their characterization was made according to the farming practices, performance, their frequency in the townships and in the ethnic groups. The results obtained allowed the identification of four growing systems of Organic Cotton: The system of mechanized cultivation with animal traction that does not use new technological packages (S1); the system of manual culture which uses vegetables like previous cropping (S2); the system of mechanized cultivation with animal traction which uses new technological packages (S3) and the system of mechanized cultivation with animal traction which uses new technological packages but continues with the practice of clearing with fire (S4). These systems are distinguished primarily by the variables such as the preparation of the soil, the rotation, the previous cropping, the organic fertilizer, plants insecticides, plants traps and associated cultures. All the producers investigated at Glazoué, Kandi and Sinendé practised respectively the growing systems S2, S1 and S4. Banikoara has, 96,67\% of the growers are the system S3 against 3.33\% for the system S1. The producers of Ouassa-Péhunco practised at $77.78 \%$ the system S3 against $22.22 \%$ for the system S1. Respondents of the ethnic groups Adja, Fon and Mahi practised at 100\% the system S2. However all Haoussa and Monkolé practised the system S1. Gando, Peulh and Bariba did not practise a system specifically. The system S2 obtained the highest average yield $(885,80$ $\pm 36.3 \mathrm{~kg} / \mathrm{ha}$ ). In conclusion, the growing systems vary according to the Common and the ethnic groups. The System $\mathrm{S} 1$ was more practised by the producers.
\end{abstract}

(C) 2018 International Formulae Group. All rights reserved.

Keywords: Growing systems, organic cotton, farming practices, Benin, mechanized ploughing.

\section{INTRODUCTION}

Le coton biologique est cultivé depuis plus d'une vingtaine d'années dans plusieurs pays du monde, en réponse à des préoccupations environnementales et sociales des consommateurs des pays du Nord (Haynes, 2006). Le coton biologique était cultivé sur une superficie de 220765 ha par près de 148000 agriculteurs recensés en 2013-2014. Cela représentait $0,7 \%$ des surfaces mondiales de coton (Le Douarin, 2016). Après avoir connu une baisse pendant trois campagnes, la production de coton-fibre biologique a amorcé une progression de $10 \%$ en 2013-2014, atteignant 116974 tonnes (Le Douarin, 2016). Les raisons de cette croissance sont notamment l'augmentation de la demande, en particulier en Europe, et une hausse de la productivité en Inde. Selon les estimations de Textile Exchange, la production de coton biologique a progressé de 15 à $20 \%$ en 2014-2015 (Le
Douarin, 2016). Les principaux pays producteurs de coton biologique sont l'Inde (74\% de la production mondiale en 2013-2014), la Chine (10\%), la Turquie (7\%), la Tanzanie (3\%) et les Etats-Unis (2\%) (Le Douarin, 2016).

En Afrique, la production du coton biologique a démarré dans quatre pays : le Bénin, le Burkina -Faso, le Mali et le Sénégal en 2002 avec le projet Helvetas, appuyé notamment par la coopération suisse (SECO) et une ONG néerlandaise (ICCO) jusqu'en 2007, pour un total d'environ 3 millions d'Euros, en partenariat avec des industriels du textile et de la grande distribution. En 2006 la production a atteint 406 tonnes de coton-graine biologique, pour 2445 ha et 4677 producteurs africains (AFD et al., 2008). Au cours de la campagne 2010-2011, un total de 11749 tonnes de cotongraine biologique et bio- équitable a été produit en Afrique (Tovignan, 2012). 
Au Bénin en particulier, la promotion de la production et la commercialisation du coton biologique certifié remonte à 1996 avec l'Organisation Béninoise pour la Promotion de l'Agriculture Biologique (OBEPAB). Cette organisation intervenait dans les Communes de Djidja (Département du Zou) et Glazoué (Département des Collines) situées au Bénin. Le Projet d'Appui au Développement de la Commune de Kandi (PADEC) intervenait aussi pour la même cause dans cette Commune située au Nord du Bénin. Aujourd'hui, plusieurs Communes sont impliquées dans la production du coton biologique (Assogba et al., 2013). Parmi ces Communes figurent Banikoara, Kandi, Gogounou, Sinendé, Ouassa-Péhunco, Kouandé, Matéri, Tanguiéta, Glazoué et Djidja. Mais, les impacts négatifs de l'exploitation incontrôlée de ces écosystèmes peuvent se traduire par une baisse des rendements, ce qui s'aggrave au fur et à mesure de l'intensification du réchauffement du climat (Abdoul Habou et al., 2016). Or, l'agriculture moderne est essentiellement dépendante d'intrants inorganiques pour une production intensive (FAO, 2003 ; Bouet et al., 2012; Tunya et al., 2014).

La présente étude vise à faire la typologie des systèmes de culture du coton biologique au Bénin. De façon spécifique, il s'agit d'identifier les systèmes de culture pratiqués par les producteurs du coton biologique et de caractériser ces systèmes. La connaissance des typologies des exploitations agricoles permet de définir les groupes cibles concernés, de comparer ces exploitations et de juger de leur fonctionnement, pour des interventions efficaces (Mbetid-Bessane et al., 2002). Cette typologie permettra de fournir aux décideurs, une image des différentes activités agricoles locales en vue d'une orientation des actions de développement (Arbelot et al., 1997).

\section{MATERIEL ET METHODES Milieu d'étude}

La présente étude a été menée dans cinq Communes au Nord et au Centre du Bénin.
Il s'agit des communes de Kandi, Banikoara, Sinendé, Ouassa-Péhunco et Glazoué (Figure 1). Ces Communes font partie des grandes zones de production du coton biologique au Bénin (Hougni et al., 2012). Elles ont été identifiées sur la base des données actuelles de production cotonnière grâce aux informations recueillies lors de la phase exploratoire auprès des structures d'encadrement des cotonculteurs biologiques tels que l'OBEPAB, l'Association des Femmes Vaillantes et Actives (AFVA/ONG), l'Union des Producteurs de Coton Biologique (UPC-Bio), Helvetas. Le choix des villages a été fait de manière raisonnée et a tenu compte non seulement de la représentativité en matière de production de coton biologique mais aussi de la présence dans les villages, d'un agent d'encadrement ou d'un représentant des producteurs de coton biologique reconnu par les structures d'encadrement pour faciliter la collaboration avec les producteurs.

\section{Méthode \\ Collecte des données}

Les travaux de recherche ont consisté à réaliser une enquête auprès des parties prenantes de la production du coton-graine biologique à Kandi, Banikoara, Sinendé, Ouassa-Péhunco, et Glazoué. Des entretiens de focus groups ont été organisés aussi pour la collecte des données en milieu réel. Pour ce faire, une phase exploratoire a eu lieu pour prendre contact avec les actifs agricoles des zones de production du coton-graine biologique, les agents techniques d'encadrement, les personnes ressources, les services compétents intervenant dans le secteur et les autorités locales. Ensuite, une pré-enquête a été effectuée pour tester les outils de collecte des données afin de les réajuster. Enfin, la phase d'enquête proprement dite a permis de collecter les données sur le terrain.

La taille de l'échantillon considéré $\mathrm{n}=176$ (Tableau 1) a été déterminée en utilisant l'approximation normale de la loi binomiale (Dagnelie, 1998) : 
$n=\mathrm{U}^{2} 1-\alpha / 2 \frac{P(1-P)}{d^{2}}$. Dans cette formule, $\mathrm{p}$

est la proportion de producteurs de coton biologique de la Commune ; $\mathrm{U}_{1-\alpha / 2}$ est la valeur de la loi normale liée à la valeur de probabilité $1-\alpha / 2$ avec $\alpha=5 \%$ soit 1,96 ; d est la marge d'erreur de l'estimation qui est fixée à $5 \%$.

Dans chaque village, le choix des producteurs de coton-graine biologique se fait sur la base d'un tri au hasard parmi ceux reconnus par les responsables de coopératives de producteurs de coton-graine biologique. De plus, deux focus groups ont été réalisés dans chaque zone au sein de laquelle, les meilleurs villages produisant le coton-graine biologique sont priorisés. Ces focus groups ont été réalisés avec les producteurs de coton-graine biologique, les agents d'encadrement et les agents des ONG qui interviennent dans le domaine. Enfin, des entretiens semi-structurés ont été effectués avec les personnes ressources exerçant dans les structures qui interviennent dans la production du coton-graine biologique au Bénin.

Le questionnaire a porté sur les grandes lignes telles que (1) accès à la terre et son statut, (2) association et rotation de cultures, (3) ancienneté dans la production du coton biologique, (4) choix de semence et semis du coton biologique, (5) opérations d'entretien des champs, (6) gestion de la fertilité du sol, (7) gestion des ravageurs et des maladies, (8) récolte, (9) commercialisation puis (10) l'identité des producteurs et leurs caractéristiques socio-culturelles.

\section{Méthode d'analyse statistique des données}

Pour faire la typologie des systèmes de culture de coton biologique, le logiciel $\mathrm{R}$, version 3.3.2 (2016) a été utilisé pour effectuer les analyses suivantes: (1) une Analyse des Correspondances Multiples (ACM) avec le package FactoMineR qui a permis d'obtenir une représentation des systèmes de culture de chaque enquêté sous forme de projections sur des plans définis par des axes factoriels; (2) une Classification Ascendante Hiérarchique $(\mathrm{CAH})$ réalisée sur les coordonnées des systèmes de culture de chaque enquêté dans le plan factoriel formé par les deux premières dimensions de l'ACM afin de définir les systèmes de culture ; (3) des fréquences ont été calculées pour les systèmes retenus par Commune et par ethnie afin de voir si un système de culture n'est pas propre à une Commune ou est une connaissance traditionnelle d'une ethnie. Ces fréquences ont été présentées sous forme de graphiques avec la fonction barplot; (4) une Analyse en Composantes Principales (ACP) a été réalisée sur les fréquences calculées en mettant en colonne les Communes et les ethnies afin de voir le lien entre eux selon leurs proportions pour chaque système de culture. L'ACP est une méthode d'analyse statistique multivariée dont l'objectif est de réduire le nombre de variables. Elle permet d'établir une similarité entre les individus, de chercher des groupes d'individus homogènes, et de mettre en évidence une typologie d'individus. En effet, l'ACP est souvent utilisée lorsqu'il est évident que les variables de l'étude sont corrélées entre elles (Aldenderfer et Blashfield, 1984). Cette méthode a été utilisée pour réduire le nombre de variables à utiliser dans le but d'éliminer les effets dus aux différences de moyennes et au choix des unités. Les nouvelles variables obtenues appelées facteurs ou composantes principales ne présentent aucune corrélation entre elles. Le nombre de composantes principales qu'il est possible d'extraire a été déterminé en utilisant le critère de Kaiser (Kaiser, 1960) ; (5) les rendements moyens en coton-graine ont été calculés pour chaque système de culture et présentés sous forme de graphique avec la fonction barplot. Le test de Kruskall Wallis a été réalisé pour comparer les rendements moyens en coton-graine des systèmes. 


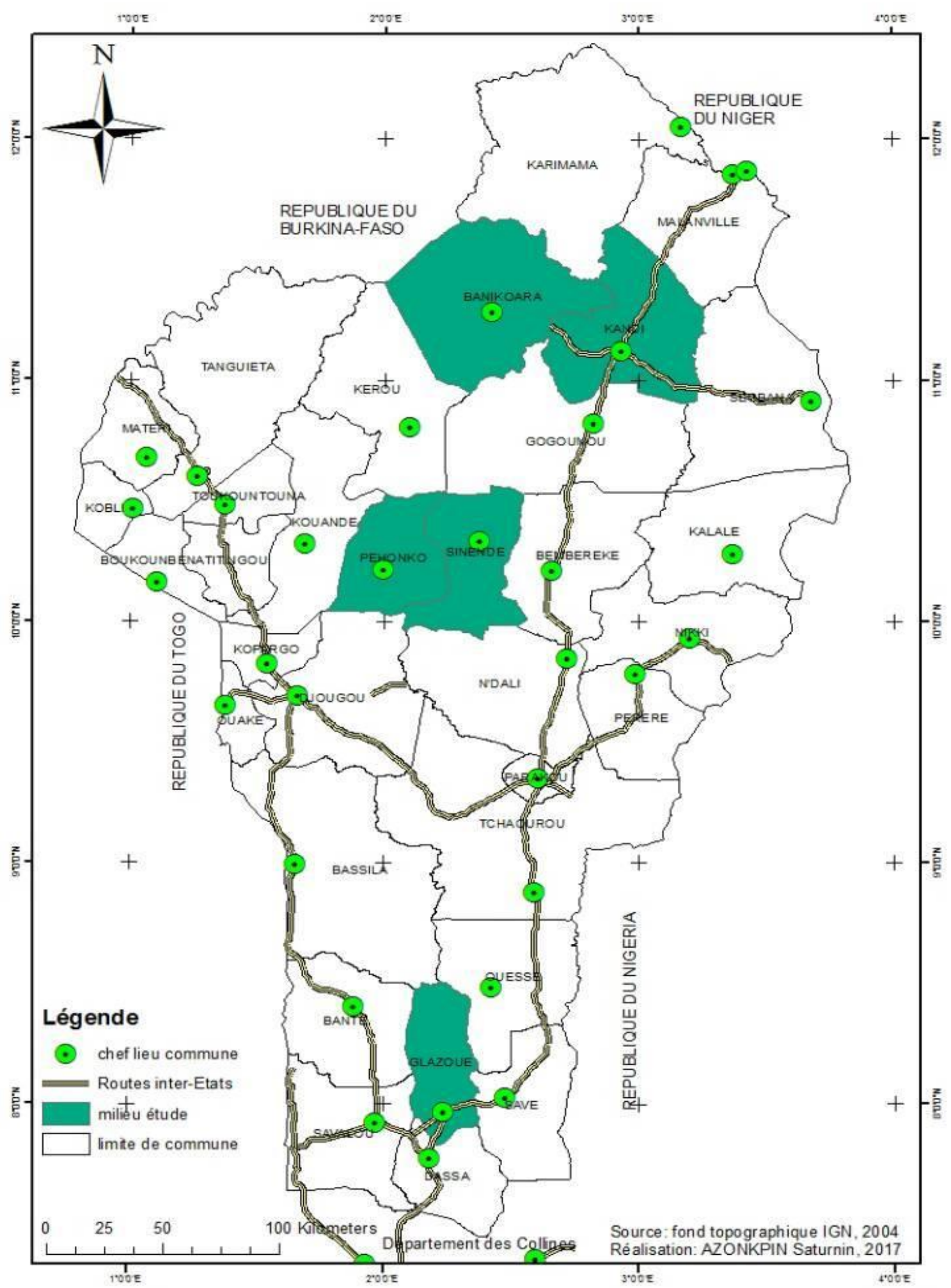

Figure 1: Carte du Bénin montrant les Communes dans lesquelles le travail a été réalisé. 
Tableau 1 : Nombre de producteurs enquêtés par Commune pour cette étude.

\begin{tabular}{lcc}
\hline COMMUNES & $\begin{array}{c}\text { EFFECTIF DES } \\
\text { PRODUCTEURS }\end{array}$ & $\begin{array}{c}\text { NOMBRE DE PRODUCTEURS } \\
\text { ENQUETES }\end{array}$ \\
& EN 2016 & 61 \\
\hline Kandi & 1500 & 30 \\
Banikoara & 200 & 25 \\
Sinendé & 191 & 10 \\
Ouassa-Péhunco & 18 & 50 \\
Glazoué & 500 & $\mathbf{1 7 6}$ \\
TOTAL & $\mathbf{2 4 0 9}$ & \\
\hline
\end{tabular}

\section{RESULTATS \\ Diversité des systèmes de culture du coton biologique \\ Les deux premières dimensions} obtenues après l'Analyse des Correspondances Multiples (ACM) expliquent $12,82 \%$ de la variabilité totale de l'échantillon (Figure 2). Les variables telles que l'écimage, l'utilisation des plantes pièges pour les insectes, les plantes insecticides, la rotation, l'association de culture, la préparation du terrain et les précédents culturaux sont bien expliquées par la première composante principale, qui à elle seule, représente $7,35 \%$ de la variabilité des informations collectées. $\mathrm{La}$ seconde composante principale explique plus les variables engrais organiques et aussi la préparation du terrain et les précédents culturaux.

$$
\text { La Classification Ascendante }
$$

Hiérarchique $(\mathrm{CAH})$ réalisée à partir des coordonnées des observations sur les deux dimensions retenues à partir de l'ACM a fait ressortir quatre types de systèmes de culture de coton biologique qui se distinguent plus par les variables telles que la préparation du terrain, la rotation, les précédents culturaux, les engrais organiques, les plantes insecticides, les plantes pièges, les cultures associées dans l'ordre d'importance (Tableau 2). Le système de culture $\mathrm{S} 4$ regroupe moins de producteurs avec $14,29 \%$ du total des enquêtés. Il est suivi par les systèmes de culture $\mathrm{S} 3, \mathrm{~S} 2$ et $\mathrm{S} 1$ avec respectivement $20,57 \%, 28,57 \%$ et $36,57 \%$ des enquêtés (Figure 3).

\section{Caractérisation technique des systèmes de culture du coton biologique}

Le tableau 3 présente la statistique $\mathrm{v}$ test calculée pour chaque modalité des variables qui discriminent mieux les différents systèmes de culture. Le système de culture du coton biologique $\mathrm{S} 1$ dénommé système de culture mécanisée avec traction animale qui n'utilise pas de nouveaux paquets technologiques, regroupe $95 \%$ des producteurs enquêtés qui utilisent le labour avec les bœufs de trait pour la préparation du sol et $89,06 \%$ des producteurs classés dans ce système utilisent ce mode de préparation du sol. Ce système regroupe également $64,65 \%$ des enquêtés qui mettent en œuvre la rotation et $100 \%$ des individus de ce système la pratiquent. Les producteurs de ce système font une seule récolte, utilisent la bouse de bovin plus fumier de ferme ou la bouse de vache plus tourteau de palmiste pour enrichir le sol. Aussi traitent-ils les plants de coton avec le filtrat des feuilles du neem (Azadirachta indica Juss.) plus piment plus du savon indigène.

Le système de culture S2 dénommé système de culture manuelle qui utilise les légumineuses comme précédent cultural compte, en son sein, les producteurs qui font le défrichement sans brûlis plus labour manuel pour une proportion de $87,72 \%$ des enquêtés qui utilisent ce mode et appartiennent à ce système. Une proportion de $83,33 \%$ et $94,12 \%$ respectivement des producteurs enquêtés appartiennent à ce système ayant pour 
précédent cultural les légumineuses et la monoculture.

Les variables qui discriminent plus le système de culture S3 dénommé système de culture mécanisée avec traction animale qui utilise de nouveaux paquets technologiques, sont la préparation du terrain (défrichement plus labour avec bœuf de trait pour $88,86 \%$ des enquêtés de cette modalité dans le système), l'utilisation des plantes pièges (pour $54,10 \%$ des enquêtés de cette modalité dans ce système) et les engrais organiques (bouse de vache plus compost pour 100\% de cette modalité dans ce système).

Le système de culture S4 dénommé système de culture mécanisée avec traction animale qui utilise de nouveaux paquets technologiques mais qui continue avec la pratique de défrichement avec brûlis, regroupe les producteurs qui pratiquent l'association et la rotation $(92,59 \%$ des enquêtés de cette modalité dans le système), préparent leur sol en faisant le défrichement avec brûlis plus labour avec les bœufs de trait $(90,92 \%$ des enquêtés de cette modalité dans ce système), associent plus le maïs ou le niébé au coton (100\% des enquêtés de cette modalité dans ce système).

On remarque que les systèmes de culture $\mathrm{S} 1$ et $\mathrm{S} 2$ regroupent des producteurs qui n'utilisent pas de plantes pièges et ne font pas l'écimage. Par contre, les systèmes de culture S3 et S4 regroupent ceux qui utilisent les plantes pièges pour la gestion des insectes et font l'écimage. On observe également que pour la préparation du sol, tous les quatre systèmes ont leurs particularités pour cette variable. Le système S1 n'utilise que le labour avec bœuf de trait, les systèmes $\mathrm{S} 2$ et $\mathrm{S} 3$ font tous le défrichement sans brûlis mais respectivement plus labour manuel et labour avec bœuf de trait. Pour ce qui est du système $S 4$, les producteurs de cette classe font le défrichement avec brûlis plus labour avec bœuf de trait. De même pour la variable rotation, la rotation, la monoculture, la rotation plus jachère et l'association plus la rotation sont les modalités qui caractérisent respectivement les systèmes de culture $S 1, S 2$, $\mathrm{S} 3$ et S4. La lutte contre les insectes pour tous les systèmes de culture est constituée du filtrat de feuille de neem, seuls les produits additifs sont différents. La bouse de bovin est utilisée dans les trois premiers systèmes plus d'autres engrais organiques pour la fertilisation des champs.

\section{Caractéristiques socio-ethniques des producteurs dans chaque système de culture du coton biologique}

La caractérisation des producteurs de chaque système selon les Communes a montré que $100 \%$ de producteurs enquêtés dans les Communes de Glazoué, Kandi et Sinendé ont pratiqué respectivement les systèmes de culture S2, S1 et S4 (Figure 4). Dans la Commune de Banikoara, 96,67\% des producteurs enquêtés ont pratiqué le système de culture $\mathrm{S} 3$ contre $3,33 \%$ pour le système $\mathrm{S} 1$. Les producteurs du coton biologique de la Commune de OuassaPéhunco ont pratiqué à $77,78 \%$ le système $\mathrm{S} 3$ contre $22,22 \%$ pour le système S1. La Figure 5 situe sur une carte, la répartition des systèmes de culture dans les Communes.

Les enquêtés des ethnies Adja, Fon et Mahi ont pratiqué à $100 \%$ le système de culture S2. Par contre, $100 \%$ des enquêtés des Haoussa et Monkolé ont pratiqué le système S1. Les Gando, Peulh et Bariba n'ont pas un système de prédilection mais ils pratiquent plusieurs systèmes de culture (Figure 6).

L'analyse en composantes principales (ACP) sur la fréquence des producteurs dans les systèmes selon les caractéristiques socioethniques explique $73,04 \%$ avec les deux premières composantes principales d'informations constituées. La première composante principale explique mieux le système $\mathrm{S} 2$ avec un regroupement des ethnies Fon, Adja et Mahi dans ce système. Le système S2 est plus pratiqué dans la Commune de Glazoué. La deuxième composante principale explique mieux les systèmes S3 et S4 qui s'opposent au système S1 sur l'axe. Les systèmes $\mathrm{S} 3$ et $\mathrm{S} 4$ sont plus pratiqués par les Peulh et les Gando. Les producteurs de Sinendé, Banikoara et Ouassa-Péhunco ont pratiqué les systèmes $\mathrm{S} 3$ et $\mathrm{S} 4$. Le système $\mathrm{S} 1$ est pratiqué plus à Kandi par les Haoussa et les Monkolé (Figure 7).

Les rendements moyens en coton-graine calculés pour chaque système placent le système S2 en tête comme système ayant la production moyenne la plus élevée $(885,80 \pm$ $36,3 \mathrm{~kg} / \mathrm{ha}$ ) et le système $\mathrm{S} 4$ comme celui qui a la production moyenne la plus faible $(734,80 \pm$ 40,20 kg/ha) (Figure 8). Le test de KruskalWallis a permis de conclure que la variation de la production moyenne entre les différents systèmes est significative $(\mathrm{P}=0,006)$. 


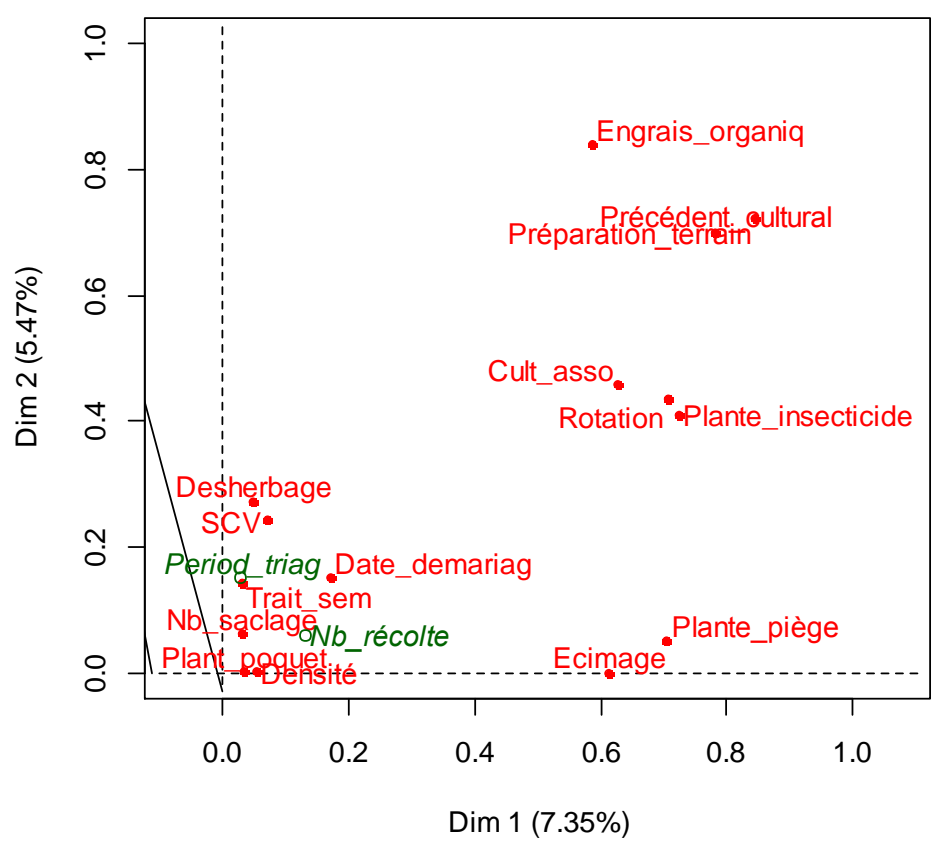

Figure 2 : Projection des variables dans le plan formé par les deux premières dimensions de l'ACM.

Tableau 2 : Classification Ascendante Hiérarchique (CAH) des coordonnées des systèmes de culture de chaque enquêté.

\begin{tabular}{lcc}
\hline \hline & P. value & df \\
\hline Préparation du terrain & 0,000 & 24 \\
Rotation & 0,000 & 24 \\
Précédent cultural & 0,000 & 33 \\
Engrais organiques & 0,000 & 45 \\
Plante insecticide & 0,000 & 48 \\
Plante piège & 0,000 & 3 \\
Culture associée & 0,000 & 27 \\
Ecimage & 0,000 & 3 \\
Désherbage & 0,000 & 3 \\
Nombres de récolte & 0,000 & 15 \\
Semis Sous Couvert Végétale & 0,000 & 3 \\
Période de triage & 0,000 & 6 \\
Date de démariage & 0,000 & 9 \\
Nombres de sarclage & 0,000 & 9 \\
Traitement de semence & 0,007 & 3 \\
\hline P. value= Valeur de la probabilité $;$ df = Degré de liberté
\end{tabular}




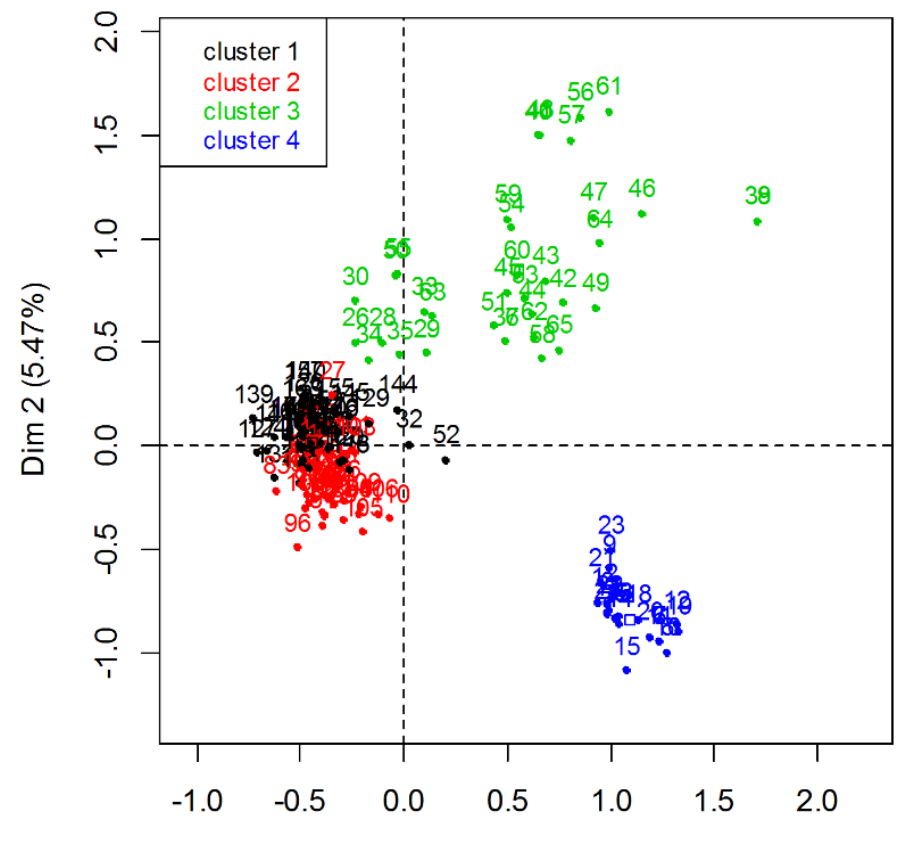

$\operatorname{Dim} 1(7.35 \%)$

Figure 3 : Projection des producteurs de coton dans le plan formé par les deux premières dimensions de l'ACM.

Tableau 3: Caractéristiques des systèmes de culture du coton biologique selon les modalités qui caractérisent mieux chaque système.

\section{Système 1}

\begin{tabular}{|c|c|c|c|c|c|}
\hline Variable/Modalité & Cla/Mod & Mod/Cla & Global & p.value & v.test \\
\hline $\begin{array}{l}\text { Préparation du terrain= Labour bouf } \\
\text { de trait }\end{array}$ & 95,00 & 89,06 & 34,09 & $<0,000$ & 12,23 \\
\hline Précédent cultural=Coton & 86,96 & 93,75 & 39,20 & $<0,000$ & 11,80 \\
\hline Rotation=Rotation & 64,65 & 100,00 & 56,25 & $<0,000$ & 9,82 \\
\hline Nombre de récolte $=1$ & 96,97 & 50,00 & 18,75 & $<0,000$ & 8,15 \\
\hline $\begin{array}{l}\text { Engrais organiques=Bouse de vache }+ \\
\text { fumier de ferme }\end{array}$ & 100,00 & 42,19 & 15,34 & $<0,000$ & 7,67 \\
\hline Ecimage $=$ Non & 55,14 & 92,19 & 60,80 & $<0,000$ & 6,79 \\
\hline $\begin{array}{l}\text { Plantes insecticides =Neen+ papaye+ } \\
\text { piment+ savon }\end{array}$ & 87,88 & 45,31 & 18,75 & $<0,000$ & 6,74 \\
\hline Plantes pièges=Non & 51,30 & 92,19 & 65,34 & $<0,000$ & 5,95 \\
\hline $\begin{array}{l}\text { Engrais organiques=Bouse de vache+ } \\
\text { tourteau de palmiste+ fumier de ferme }\end{array}$ & 100,00 & 25,00 & 9,09 & $<0,000$ & 5,58 \\
\hline
\end{tabular}

Système 2 


\begin{tabular}{|c|c|c|c|c|c|}
\hline Variable/Modalité & Cla/Mod & Mod/Cla & Global & p.value & v.test \\
\hline $\begin{array}{l}\text { Préparation du terrain =Défrichement sans } \\
\text { brûlis + labour manuel }\end{array}$ & 87,72 & 98,04 & 32,39 & $<0,000$ & 12,35 \\
\hline Précédent cultural=Culture légumineuse & 83,33 & 78,43 & 27,27 & $<0,000$ & 9,54 \\
\hline Rotation=Monoculture & 94,12 & 62,75 & 19,32 & $<0,000$ & 9,04 \\
\hline $\begin{array}{l}\text { Plantes insecticides }=\text { Neem }+ \text { papaye }+ \\
\text { piment }+ \text { agribiopesticide }\end{array}$ & 87,18 & 66,67 & 22,16 & $<0,000$ & 8,77 \\
\hline Plantes pièges $=$ Non & 44,35 & 100,00 & 65,34 & $<0,000$ & 7,02 \\
\hline $\begin{array}{l}\text { Engrais organiques }=\text { Bouse de vache }+ \\
\text { tourteau palmiste }\end{array}$ & 70,00 & 54,90 & 22,73 & $<0,000$ & 6,19 \\
\hline Engrais organiques=Tourteau palmiste & 59,46 & 43,14 & 21,02 & $<0,000$ & 4,36 \\
\hline Nombre récolte $=2$ & 36,57 & 96,08 & 76,14 & $<0,000$ & 4,30 \\
\hline Désherbage $=$ Sarclage et sarclobutage & 34,72 & 98,04 & 81,82 & $<0,000$ & 3,92 \\
\hline Culture associée $=$ Rien & 34,25 & 98,04 & 82,95 & $<0,000$ & 3,73 \\
\hline \multicolumn{6}{|c|}{ Système 3} \\
\hline Variable/Modalité & Cla/Mod & Mod/Cla & Global & p.value & v.test \\
\hline $\begin{array}{l}\text { Préparation du terrain =Défrichement sans } \\
\text { brûlis + labour bœuf de trait }\end{array}$ & 88,46 & 63,89 & 14,77 & $<0,000$ & 8,28 \\
\hline Plantes pièges=oui & 54,10 & 91,67 & 34,66 & $<0,000$ & 8,00 \\
\hline $\begin{array}{l}\text { Engrais organiques }=\text { Bouse de vache }+ \\
\text { compost }\end{array}$ & 100,00 & 44,44 & 9,09 & $<0,000$ & 7,27 \\
\hline Désherbage $=$ Sarclage & 71,88 & 63,89 & 18,18 & $<0,000$ & 7,16 \\
\hline Semis sous Couvert Végétal =oui & 66,67 & 50,00 & 15,34 & $<0,000$ & 5,74 \\
\hline $\begin{array}{l}\text { Précédent cultural=Culture de céréale ou } \\
\text { tubercule }\end{array}$ & 100,00 & 25,00 & 5,11 & $<0,000$ & 5,15 \\
\hline $\begin{array}{l}\text { Période triage }=\text { Au cours de la récolte et } \\
\text { après la récolte }\end{array}$ & 100,00 & 22,22 & 4,55 & $<0,000$ & 4,80 \\
\hline Ecimage $=$ Oui & 37,68 & 72,22 & 39,20 & $<0,000$ & 4,45 \\
\hline Nombre de sarclage $=4$ à 5 & 60,00 & 33,33 & 11,36 & $<0,000$ & 4,09 \\
\hline Rotation $=$ Rotation - Jachère & 100,00 & 16,67 & 3,41 & $<0,000$ & 4,05 \\
\hline \multicolumn{6}{|c|}{ Système 4} \\
\hline Variable/Modalité & Cla/Mod & Mod/Cla & Global & p.value & v.test \\
\hline Rotation =Association-Rotation & 92,59 & 100,00 & 15,34 & $<0,000$ & 11,04 \\
\hline $\begin{array}{l}\text { Préparation du terrain =Défrichement avec } \\
\text { brûlis + labour bœuf de trait }\end{array}$ & 90,91 & 80,00 & 12,50 & $<0,000$ & 9,08 \\
\hline Cultures associées=Niébé, Maïs & 100,00 & 56,00 & 7,95 & $<0,000$ & 7,63 \\
\hline Plantes insecticides $=$ Neem + piment + ail & 48,94 & 92,00 & 26,70 & $<0,000$ & 7,45 \\
\hline Ecimage $=$ Oui & 36,23 & 100,00 & 39,20 & $<0,000$ & 6,99 \\
\hline Plantes pièges $=$ Oui & 37,70 & 92,00 & 34,66 & $<0,000$ & 6,38 \\
\hline Précédent cultural=Jachère & 70,00 & 56,00 & 11,36 & $<0,000$ & 6,18 \\
\hline $\begin{array}{l}\text { Engrais organiques }=\text { Tourteau palmiste }+ \\
\text { fumier de ferme }\end{array}$ & 100,00 & 36,00 & 5,11 & $<0,000$ & 5,83 \\
\hline Date de démariage $=21$ jours & 25,26 & 96,00 & 53,98 & $<0,000$ & 4,86 \\
\hline Cultures associées=Maïs, tomate & 100,00 & 20,00 & 2,84 & $<0,000$ & 4,11 \\
\hline
\end{tabular}

Clas/Mod= Proportion des enquêtés qui ont spécifié cette modalité dans ce système par rapport à l'ensemble des enquêtés qui ont spécifié cette modalité; Mod/Clas= Proportion des enquêtés qui ont spécifié cette modalité dans ce système par rapport à l'ensemble des individus de ce système; Global= Proportion des enquêtés qui ont spécifié cette modalité dans ce système par rapport à l'ensemble des enquêtés; $\mathbf{P}$. value= Valeur de la probabilité. 
S. AZONKPIN et al. / Int. J. Biol. Chem. Sci. 12(4): 1688-1704, 2018

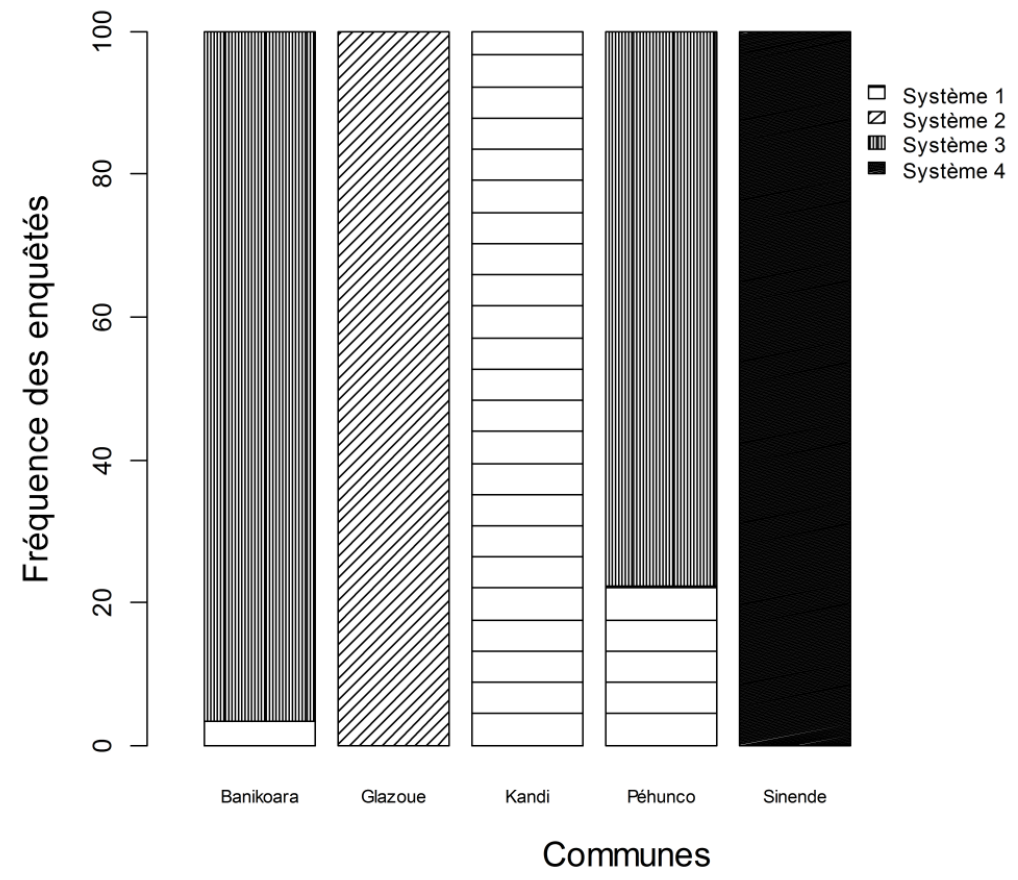

Figure 4 : Caractérisation des producteurs de chaque système selon les Communes.

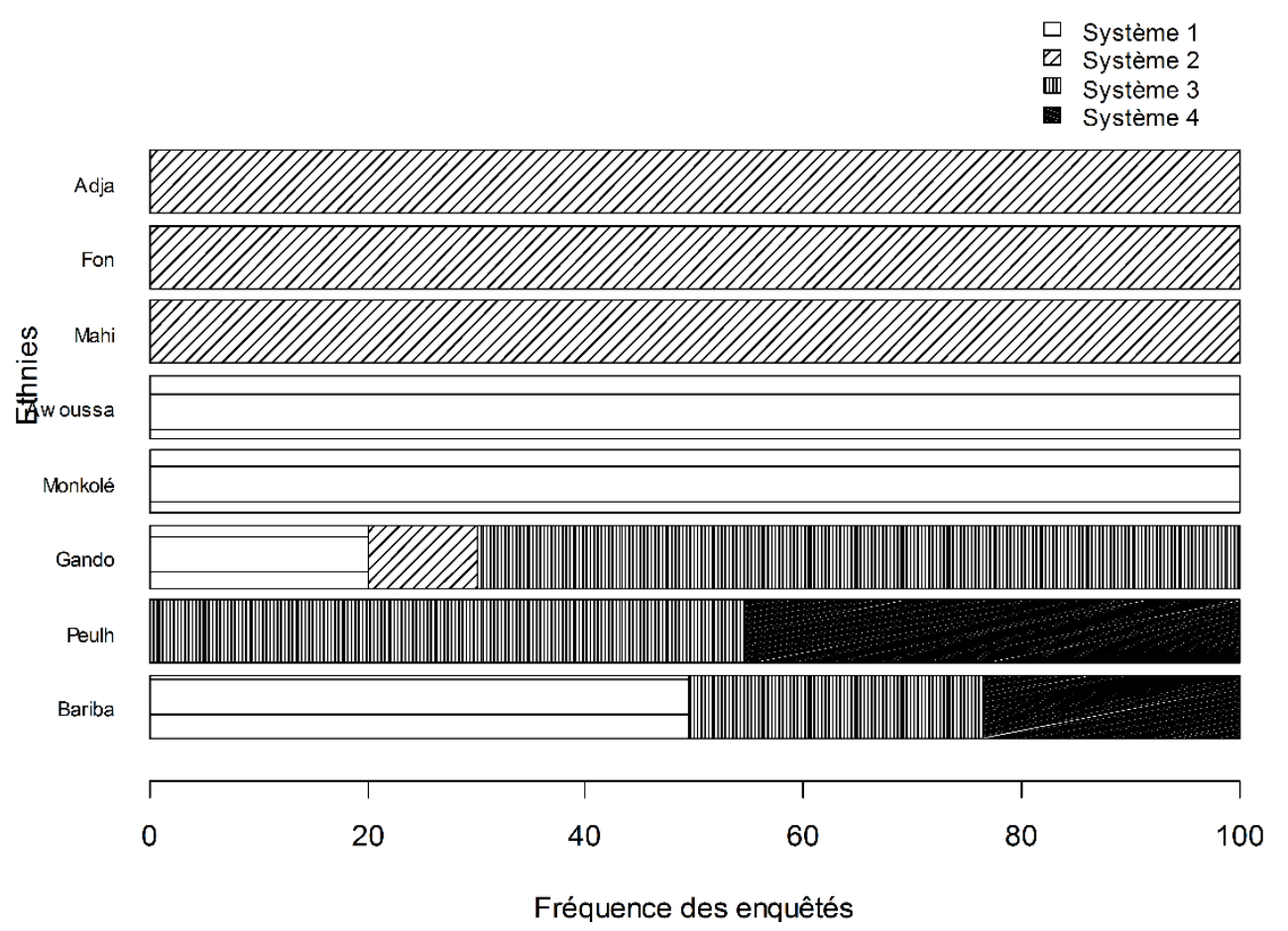

Figure 6 : Caractérisation des producteurs de chaque système selon les ethnies. 


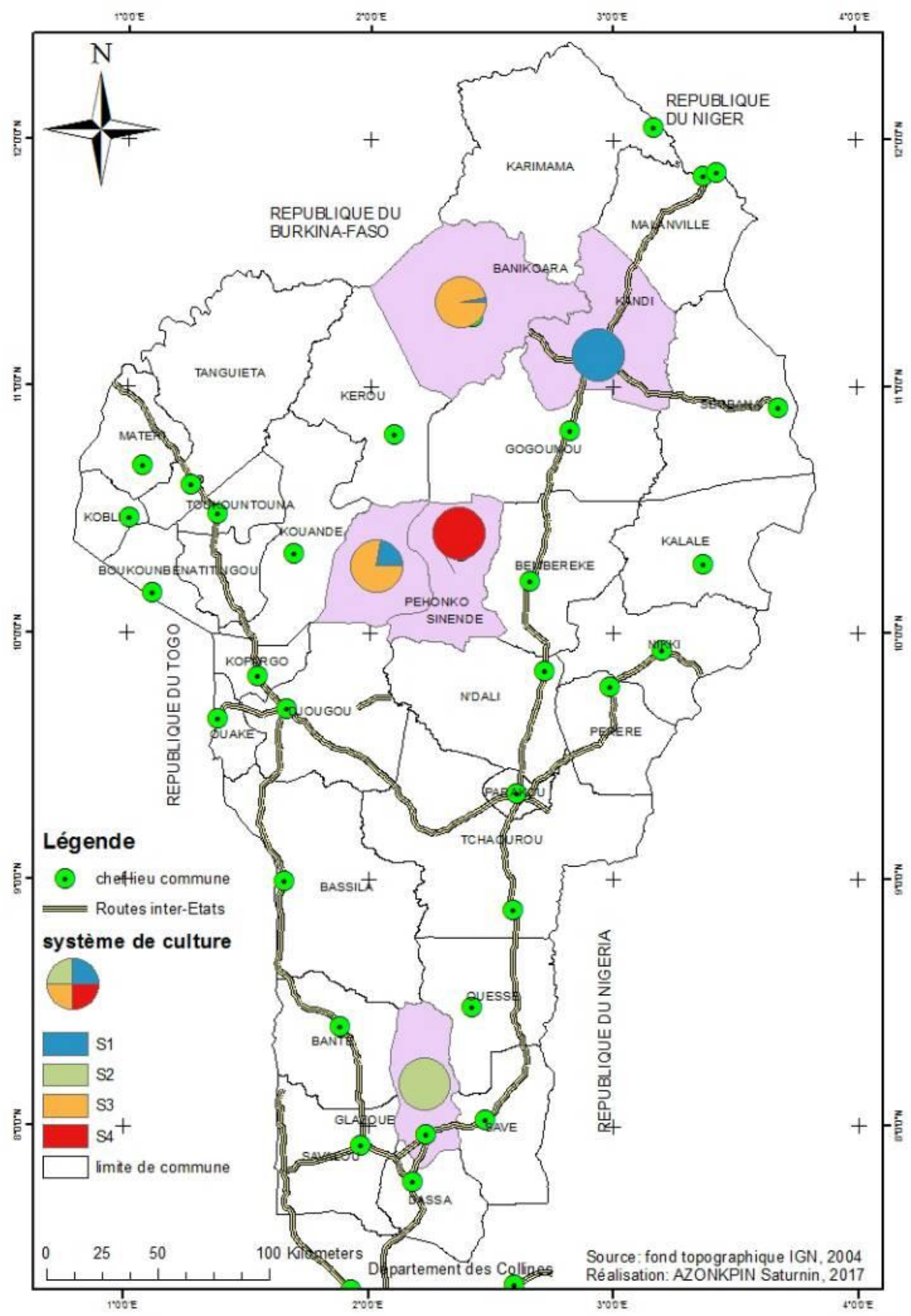

Figure 5 : Carte indiquant les systèmes de culture de coton biologique dans les Communes. 


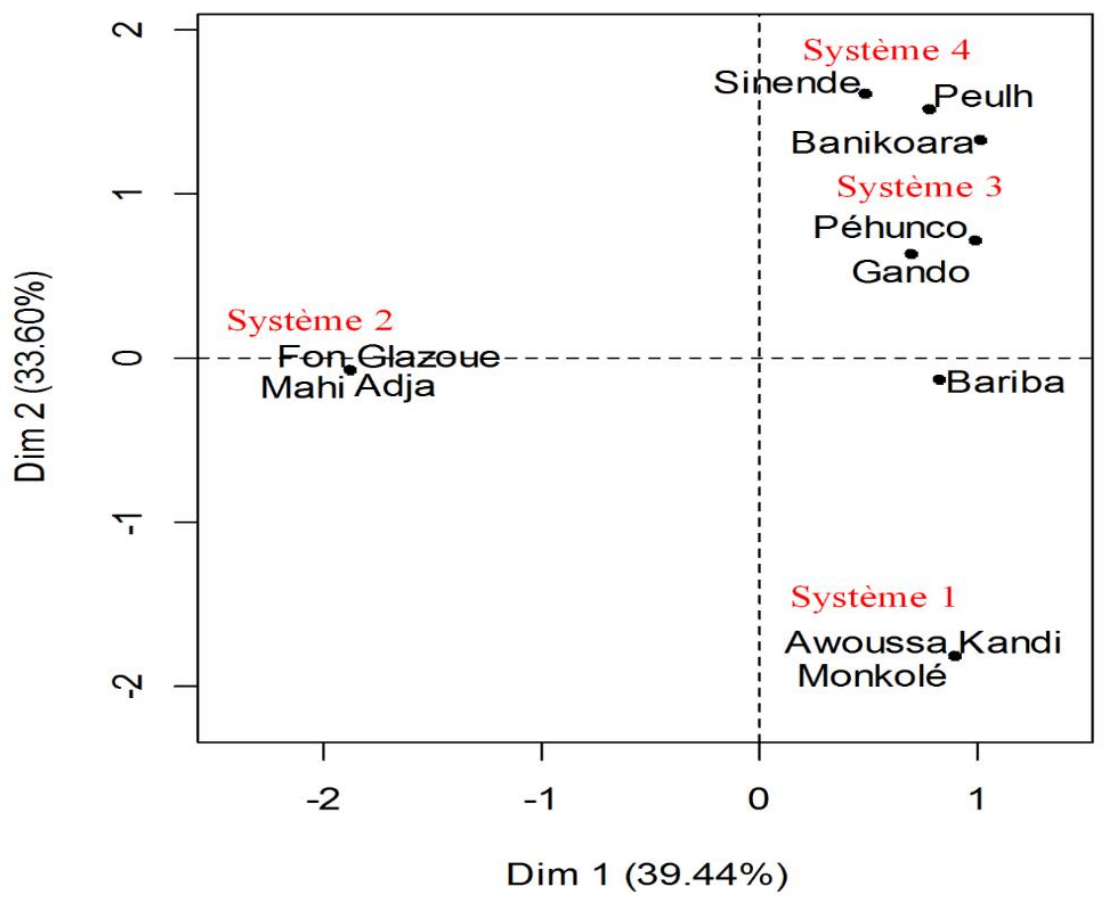

Figure 7 : Projection des individus et des variables dans les deux premières composantes Principales.

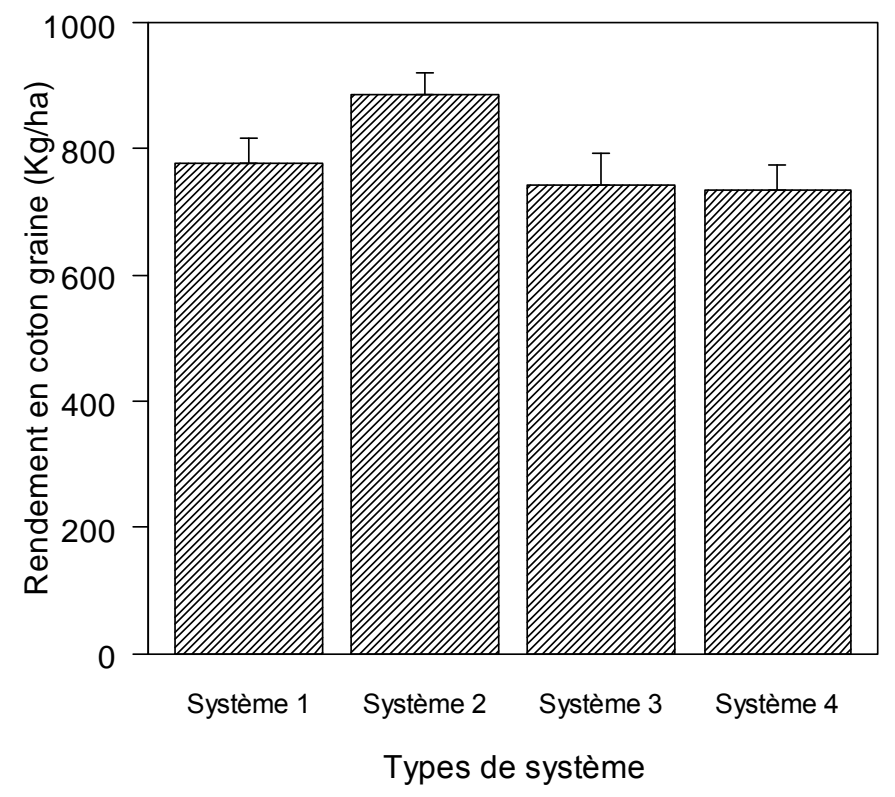

Figure 8 : Rendement moyen en coton-graine par système de culture. 


\section{DISCUSSION}

Le système de culture est l'ensemble des modalités mises en œuvre sur des parcelles traitées de manière identique. Chaque système de culture se définit par la nature des cultures, leur ordre de succession et les itinéraires techniques appliqués à ces différentes cultures, ce qui inclut le choix des variétés pour les cultures retenues (Sebillotte, 1976).

La typologie des systèmes de culture pratiqués par les cotonculteurs biologiques du Bénin a révélé quatre types de systèmes de culture de coton biologique qui se distinguent principalement par les variables telles que la préparation $\mathrm{du}$ terrain, la rotation, les précédents culturaux, l'engrais organique utilisé, les plantes insecticides, les plantes pièges utilisées, les cultures associées par ordre d'importance. Ces résultats corroborent partiellement ceux d'Allagbe et al. (2014) qui ont révélé que les variables les plus discriminantes des systèmes de culture à base de cotonculture sont la formation ou non des producteurs, la succession du maïs et de l'igname dans la rotation avec le coton, la place de l'igname en tête de rotation, la place du sorgho en tête de rotation, la superficie de coton emblavée, le type de labour (avec bœuf de trait), la quantité de NPK, de semences, d'engrais organique et la quantité de cotongraine produite. La rotation des cultures, le type de labour et les intrants agricoles influencent fortement les systèmes de culture à base de cotonculture. Tap Soba (2010) au Burkina-Faso a montré aussi que l'épandage de la fumure organique dans les champs a un impact sur la production et donc sur les performances technico-économiques du système de culture dans lequel elle est intégrée. Garane et al. (2018) ont montré l'importance de la préparation du sol dans les itinéraires techniques. Ils ont conclu que le travail minimum continu ou périodique et dans une moindre mesure le labour conventionnel continu, ont eu comme effet de favoriser la porosité et les réserves utiles du sol. Allagbe et al. (2014) ont identifié quatre systèmes de cotonculture tout comme notre étude. Mais une différence s'observe entre les systèmes de culture. En effet, leur deuxième système de culture dénommé "système de cotonculture semi-intensif avec la culture attelée", s'est retrouvé dans trois systèmes de culture $(\mathrm{S} 1, \mathrm{~S} 3$, et S4) identifiés dans notre étude à l'exception du système $\mathrm{S} 2$ de notre étude qui est manuel. Par contre, Tap Soba (2010) a identifié neuf (9) systèmes de production dans l'étude de la dynamique des systèmes de production du village de Gombèlèdougou dans la zone cotonnière de Houndé au Burkina-Faso. Il s'est basé non seulement sur la rotation où cotonmaïs a dominé mais aussi sur l'hétérogénéité des modes de conduite des cultures. Kouloumégué (2012), lors de l'évaluation des performances technico-économiques des systèmes de production à base de coton bioéquitable et Conventionnel dans la zone de Kolondièba, a dégagé trois systèmes de production: un Système de Production Conventionnelle du coton avec des traitements phytosanitaires calendaires (SPCca), un Système de Production Conventionnelle du coton avec des traitements phytosanitaires sur seuils (SPCs) et un Système de Production Biologique et Equitable (SPBE). Il a retenu ces systèmes en fonction de la logique du fonctionnement des producteurs (centrée sur l'autosubsistance ou la vente de leurs productions). Les agriculteurs choisissent de minimiser les risques de leur production ou de maximiser les profits qu'ils en espèrent en produisant l'un ou l'autre type de coton. Au Bénin, Abou et al. (2018) ont identifié également quatre (4) types de systèmes de production sur les sites d'aménagements hydroagricoles: le système de production pluviale, les systèmes de production irriguée, les systèmes de production des cultures de décrue et les systèmes de production de la pêche continentale.

Les producteurs de coton biologique enquêtés dans la Commune de Glazoué pratiquent le système de culture S2. Ces producteurs sont encadrés par l'OBEPAB. Allagbe et al. (2014) ont montré que le système de cotonculture avec la culture de l'igname en tête de rotation était prédominant dans la zone cotonnière du Centre. Dans la Commune de Kandi, c'est le système de culture S1 qui a dominé tandis qu'à Banikoara et Ouassa- 
Péhunco, le système de culture S3 a dominé dans notre étude. Ceci serait dû au fait que les producteurs de ces deux Communes sont encadrés respectivement par AFVA-ONG et UPC-bio qui sont tous appuyés par Helvetas tandis que ceux de Kandi sont encadrés par L'OBEPAB. A Sinendé, le système de culture S4 a été prépondérant. Les producteurs de cette Commune sont encadrés par l'OBEPAB. Les systèmes de culture pratiqués par les producteurs dans les Communes seraient influencés par l'encadrement. Ces trois systèmes de culture (S1, S3 et S4) ont été pratiqués par des producteurs de coton biologique qui utilisent les bœufs de trait pour leur labour. De même, les travaux effectués par Allagbe et al. (2014) ont montré que le système de cotonculture semi-intensif avec la culture attelée se retrouvait prédominant dans la zone cotonnière du nord dont font parties les Communes de Kandi, Banikoara, Sinendé et Ouassa-Péhunco.

Les enquêtés des ethnies Adja, Fon et Mahi pratiquent à $100 \%$ le système de culture S2. Il en est de même au niveau des Haoussa et Monkolé qui pratiquent tous le système S1. Par contre, les Gando, Peulh et Bariba n'ont pas de système de culture de prédilection. Parmi les cinq Communes enquêtées, les Adja, Fon et Mahi sont rencontrés seulement à Glazoué. Ils sont encadrés par l'OBEPAB. Il en est de même à Kandi où les Haoussa et les Monkolé ont été rencontrés. Par contre, les Gando, Peulh et Bariba sont rencontrés dans plusieurs Communes telles que Kandi, Banikoara, Ouassa-Péhunco et Sinendé. Ils sont donc encadrés par plusieurs structures et de ce fait ils pratiquent plusieurs systèmes de culture.

Les rendements moyens en cotongraine calculés pour chaque système placent le système $\mathrm{S} 2$ en tête comme système ayant la production moyenne la plus élevée $(885,80 \pm$ $36,30 \mathrm{~kg} / \mathrm{ha}$ ) et le système $\mathrm{S} 4$ comme celui qui a la production moyenne la plus faible $(734,80$ $\pm 40,20 \mathrm{~kg} / \mathrm{ha}$ ). Ces résultats corroborent ceux de Houndékon (2014) qui a observé la plus forte valeur de rendement du coton biologique à Glazoué où se pratique le système de culture S2. Par contre, Kouloumégué (2012), lors de son étude sur l'évaluation des performances technico-économiques des Systèmes de production à base de coton bio-équitable et Conventionnel dans la zone de Kolondièba au Mali, a trouvé des rendements plus faibles (316 $\mathrm{kg} / \mathrm{ha}$ à $368 \mathrm{~kg} / \mathrm{ha}$ ). Allagbe et al. (2014) ont révélé que le système de cotonculture semiintensif avec la culture attelée est le système de culture le plus rentable. Ils ont conclu que la pratique de la culture attelée mettant en valeur le milieu alluvionnaire des sols présente un avantage en termes de rendement des cultures et de la facilité de travail. Cependant, la pratique de la culture attelée exige des investissements financiers qui constituent des contraintes dans un contexte de pauvreté.

\section{Conclusion}

L'étude des systèmes de culture de coton biologique a permis de disposer de connaissances scientifiques sur la typologie de ces derniers au Bénin. Quatre systèmes de culture du coton biologique ont été identifiés. Ils se distinguent plus par les variables telles que la préparation du terrain, la rotation, les précédents culturaux, les engrais organiques utilisés, les plantes insecticides utilisées, les plantes pièges utilisées, les cultures associées par ordre d'importance. Le système de culture manuelle qui utilise les légumineuses comme précédents culturaux (S2) est plus pratiqué dans la Commune de Glazoué par les ethnies Fon, Adja et Mahi. Les systèmes S3 et S4 sont plus pratiqués par les Bariba, les Peulh et les Gando dans les Communes de Sinendé, Banikoara et Ouassa-Péhunco. Le système S1 est pratiqué plus à Kandi par les Haoussa et les Monkolé. Le système $\mathrm{S} 2$ a la production moyenne la plus élevée $(885,80 \pm 36,3 \mathrm{~kg} / \mathrm{ha}) . \quad \mathrm{Le}$ développement du coton biologique au Bénin, devrait s'appuyer sur ces groupes cibles et les traits distinctifs des systèmes de cultures.

\section{CONFLIT D'INTERETS}

Les auteurs déclarent n'avoir aucun conflit d'intérêts concernant cet article.

\section{CONTRIBUTIONS DES AUTEURS}

SA, initiateur de l'article, a conçu la méthodologie, a collecté les données, a écrit le manuscrit. CDC a contribué à la conception de la méthodologie, à la rédaction et à l'amélioration du manuscrit. CEA a procédé à 
l'analyse statistique des données et leur interprétation. CCJS a participé à la collecte des données et à l'amélioration du manuscrit. MMS a contribué à la conception du questionnaire et à l'amélioration du manuscrit. DSV a participé à la collecte des données, à la rédaction et à l'amélioration du manuscrit.

\section{REMERCIEMENTS}

Nous remercions tous ceux qui ont contribué d'une manière ou d'une autre à la réalisation de ce travail, notamment les techniciens qui nous ont aidés dans la collecte des données et les producteurs de coton biologiques qui ont bien voulu se confier à nous.

\section{REFERENCES}

Abou M, Yabi I, Yolou I, Ogouwale E. 2018. Caractérisation des systèmes de production sur les sites d'aménagements hydro-agricoles dans le doublet DangboAdjohoun au Sud du Bénin. Int. J. Biol. Chem. Sci., 12(1): 462-478. DOI: https://dx.doi.org/10.4314/ijbcs.v12i1.36.

Abdoul Habou Z, Boucar MK, Adam T. 2016.

Les systèmes de productions agricoles du Niger face au changement climatique : défis et perspectives. Int. J. Biol. Chem. Sci., 10(3): 1262-1272. DOI: https://dx.doi.org/10.4314/ijbcs.v10i3.

AFD. 2008. Développement du coton équitable et bio-équitable en Afrique de l'Ouest et du Centre. 56 p.

Aldenderfer MS, Blashfield RK. 1984. Cluster Analysis, Sage University Paper series on Quantitative Applications in the Social Science, series no. 07-044. Newbury Park, Califormia: Sage Publications.

Allagbé CM, Adégbola PY, Ahoyo Adjovi NR, Komlan-Ahihou CM, Crinot GFDJCE, Hessavi PM, Djenontin AJP, Mensah GA. 2014. Evaluation socio-économique des systèmes de culture à base de cotonculture au Bénin. Etude financée par le Projet Multinational d'Appui à la Filière CotonTextile dans les quatre pays de l'Initiative Sectorielle sur le Coton (PAFICOT)Bénin. $44 \mathrm{p}$.

Arbelot B, Foucher H, Dayon JF, Missohou A. 1997. Typologie des aviculteurs dans la zone du Cap-Vert au Sénégal. Élev. Méd. Vét. Pays Trop., 50(1): 75-83.

Assogba SCG, Tossou R, Lebailly P. 2013. Influence des représentations sociales de l'environnement sur l'adoption des pratiques durables de production. Une contribution à partir du système de production de coton biologique au Bénin. Université de Liège-Gembloux Agro-BioTech. 22 p.

Bouet A, Nicaise Acho Amancho A, Sanogo S, Camara M. 2012. Effet de la fertilisation azotée et phosphorée sur le développement de la Panachure jaune en riziculture aquatique en Côte d'Ivoire. Int. J. Biol. Chem. Sci., 6(6): 4071-4079. DOI: https://dx.doi.org/10.4314/ijbcs.v6i6.17.

Dagnelie P. 1998. Théories et Modèles Statistiques, Applications Agricoles, Tome 2, $21^{\text {ème }}$ Édition, 464 pp.

FAO. 2003. Association Internationale de l'Industrie des Engrais, Les engrais et leurs applications : Institut Mondial du Phosphate : Rabat ; 76p.

Garane A, Koussao S, Traore M, Sawadogo M, Péquénio PX. 2017. Influence de l'itinéraire technique sur les propriétés physiques et hydriques du sol sous culture du blé tendre (Triticum aestivum L.) dans une rotation de «longue durée» dans la zone «non-chernozem» de la Fédération de Russie. Int. J. Biol. Chem. Sci., 11(2). 886-900.

DOI: https://dx.doi.org/10.4314/ijbcs.v11i2.28.

Haynes I. 2006. Le coton bio et/ou équitable : réel avenir ou effet de mode ? Biotechnol. Agron. Soc. Environ., 10(4), 361-371.

Hougni A, Kpadé PC, Djihinto AC. 2012. Culture biologique du cotonnier et commerce équitable du coton : Deux approches de niche. Bulletin de la Recherche Agronomique du Bénin $(B R A B)$. Numéro spécial Coton. 60-74

Houndékon V. A. 2014. Analyse comparative des systèmes de production du coton biologique et du coton conventionnel au Bénin, Journal de la Recherche Scientifique de l'Université de Lomé, 16 (3) : $13 \mathrm{p}$.

Kaiser HF. 1960. The application of electronic computers to factor analysis. Educational 
and Psychological Measurement, 20 : 141-151.

Kouloumégué D. 2012. Alternative possible à la production traditionnelle du coton en Afrique dans une perspective de développement durable ? Le cas du système de production biologique et équitable au Mali. Thèse de Docteur en Sciences agronomiques et Ingénierie biologique, Université de Liège Gembloux Agro - Bio Tech, Académie Universitaire Wallonie-Europe, Communauté Française de Belgique, 161 p.

Le Douarin S. 2016. La bio dans le monde. Les carnets de l'Agence BIO, $42 \mathrm{p}$.

Mbetid-Bessane E, Havard M, Djamen NP, Djonnewa A, Djondang K, Leroy J. 2002. Typologies des exploitations agricoles dans les savanes d'Afrique centrale. Actes du colloque, 27-31.

Sebillotte M. 1976. Jachères, système de culture, système de production : méthodologie d'étude. In : Actes des journées d'études Agronomie-sciences humaines, Institut National agronomique de Paris-Grignon, Journal d'Agriculture Tropicale et de Botanique Appliquée, 24(2-3) : 241-264.

Tap Soba A. 2010. Dynamique des systèmes de production du village de Gombèlèdougou dans la zone cotonnière de Houndé : évaluation et modélisation technicoéconomique, mémoire d'ingénieur de conception en vulgarisation agricole. $67 \mathrm{p}$.

Tovignan S. 2012. Production de fibre de coton biologique et bio-équitable : Rapport pour l'Afrique 2010-2011. Textile Exchange. 7 $\mathrm{p}$.

Tunya BA, Lelei JJ, Ouma JP. 2014. Effect of chicpea (Cicer arietinum L.) and white lupin (Lupinus albus L.) on phosphorus mobilization from Minjingu phosphate rock, soil chemical properties and plant nutrient uptake in various cropping systems. In: Innovative research and technology for global development. Proceedings 26-28 mars. Egerton University Press; 301-315. 